\title{
Publisher's Note: Particle-Hole Duality in the Lowest Landau Level [Phys. Rev. Lett. 118, 206602 (2017)]
}

Dung Xuan Nguyen, Tankut Can, and Andrey Gromov

(Q) (Received 6 February 2018; published 23 February 2018)

DOI: 10.1103/PhysRevLett.120.089903

This paper was published online on 19 May 2017 with an error in the Acknowledgments. On page 5, right-hand column, the last sentence of the Acknowledgments should read as "A. G. would like to acknowledge the support of NSF Grant No. DMR-1206648." The Acknowledgments have been corrected as of 13 February 2018. The Acknowledgments are incorrect in the printed version of the journal. 